\title{
Advanced Autonomous Network Reconfiguration System
}

\author{
Abhinish Popli ${ }^{1}$, Geetanjali Rathee ${ }^{2}$, Hemraj Saini ${ }^{3}$ \\ 1,2,3 (Department of Computer Science and Engineering, Jaypee University of Information Technology, \\ Waknaghat, Solan-173234, INDIA)
}

\begin{abstract}
The Multi-hop characteristics of wireless mesh network practices recurrent nodes or link failures triggered by several reasons i.e. vibrant obstacles, channel intrusion, and applications demands. These catastrophes cause severe performance degradations and oblige expensive physical managements for their realtime recovery. In order to overcome these limitations, the aim of this manuscript is to present an Advanced Autonomous Reconfiguration Network System (ARNS) by enabling multi-radio WMNs. Using channel and radio diversities in WMNs, in order to recover from these failures, ARNS creates necessary changes in local radio and channel assignments to cooperatively reconfigure network settings among local mesh routers. The approach is proved by showing the proper simulation results.
\end{abstract}

Keywords - WMN, QoS, Multi radio WMN, AARS, Network

\section{INTRODUCTION}

WMNs [1] are being developed vigorously and deployed extensively in diverse applications like environmental monitoring, and wireless Internet services (WIS) for metro cities etc. WIS [2] has also been growing in a variety of forms (e.g., using multi radio/channel systems) to meet the increasing capacity demands by the above-mentioned and other emerging applications. However, because of heterogeneous and disturbing wireless links conditions, preserving the efficient performance of such WMNs are still a challenging problem [3]. Like some links of a WMN (as depicted in Figure 1) experiences significant channel interference from other coexisting wireless networks. Some parts of network topology might not be capable to meet cumulative bandwidth anxieties from new mobile operators and applications [4]. Links in a convinced area (e.g., a hospital or police station) might not be competent to use some incidence channels because of spectrum decorum or regulation. Many solutions have been proposed for WMNs to recuperate from wireless link failures, but they still have many limitations as resource allocation, greedy channels and fault tolerant.

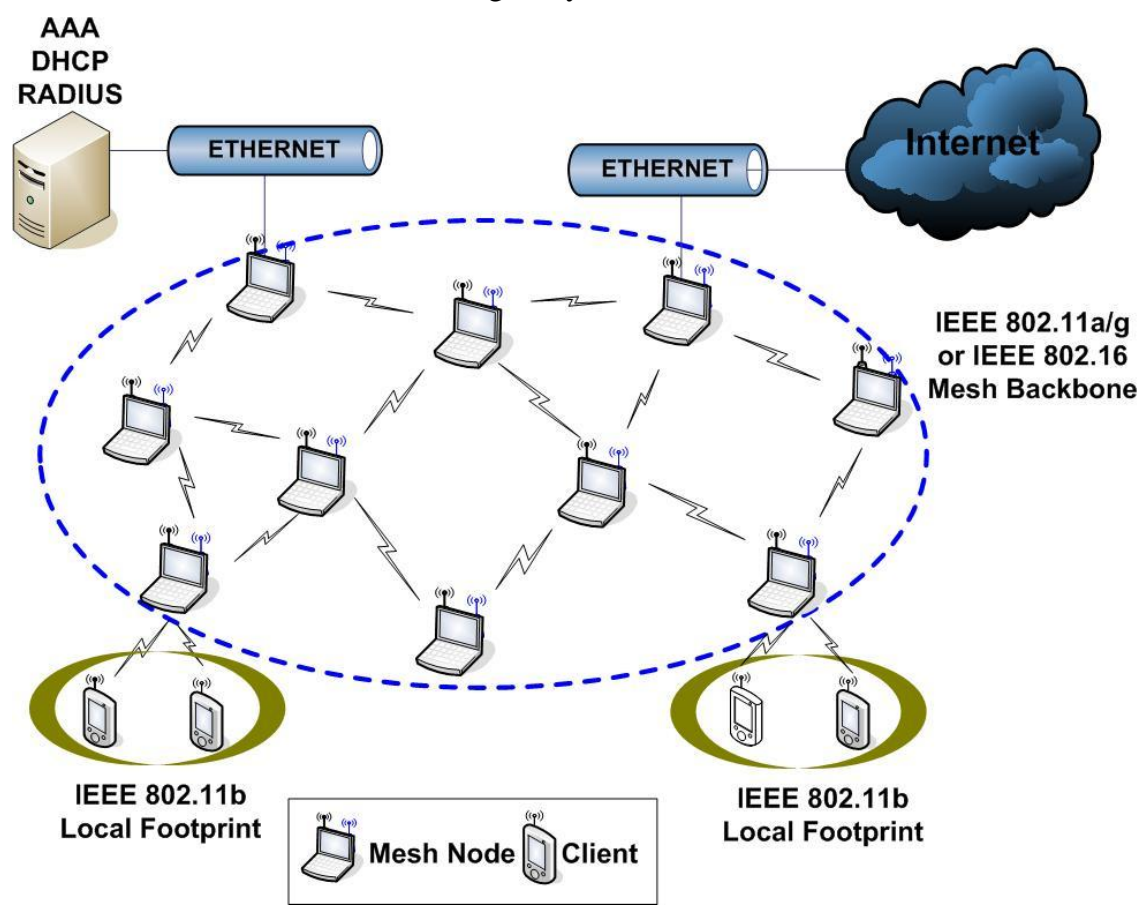

Fig. 1: Wireless Mesh Network 
To overcome the given limitations, in this paper, we have proposed an Advanced Autonomous Network Reconfiguration System (AARS) that allows a multi radio[5] WMN to autonomously reconfigure its native network settings i.e. channel, radio, and route assignment for real-time retrieval from link disasters. Mainly, AARS is fortified with a reconfiguration [6] scheduling algorithm that recognizes local configuration variations instead of global changes for the recovery, and minimizes changes in important network settings. In brief, Advanced Autonomous Network Reconfiguration System first searches for practicable local formation changes accessible around a defective zone, based on associated channel and radio configuration. Then, by considering existing network specifications as constraints, it recognizes reconfiguration [7] plans that require the least number of deviations for the vigorous network settings.

The paper is organized in five sections. Section two discusses some existing approaches on fault tolerance with their drawbacks. In section three the proposed approach (AARS) will be explained with suitable algorithm and diagram. The performance of AARS algorithm in comparison with exiting approach in terms of throughput, packet ratio and packet drop ratio is elaborated in section four. Finally in section five we conclude our paper with its future scope.

\section{RELATED WORK}

Prevailing channel assignment and development algorithms provide strategies for channel obligation during a network distribution stage. But the given existing algorithms do not deliberate the changes from preceding network settings, this type of algorithms are appropriate for periodic or static network management, but they may origin network service interruptions, and thus are inappropriate for vibrant network reconfiguration that has to covenant with recurrent local link disasters [8]. In this section we have discussed some of the existing algorithm with their drawbacks.

- Resource-allocation algorithms: Resource allocation provides the planning of resources during the initial phase of network deployment. This is useful to provide most favorable network configuration plan by requiring a global knowledge of given mesh network. The major drawback of this algorithm is that even though this approach provides an ideal network configuration strategy, but they often entail "global" configuration vagaries, which are not efficient in case of numerous local link disasters.

- Greedy channel-assignment algorithm: Greedy algorithm assigns present available channel to the node immediate after fault occurrence. Instead of having the global knowledge of the network greedy algorithm focused on defected nodes. It can reduce the necessity of network changes by altering settings of only the defective link(s). Nevertheless, this modification might not capable to grasp full perfections, which can be fulfilled by considering alignments of neighboring mesh routers in addition to the faulty links.

- Fault-tolerant routing protocols: to prevent the fault during the communication more than one channel is assigned between communicating parties. Protocols such as native multipath or rerouting can be implemented to practice the network level path variety to avoid the faulty links. However, they depend on redundant broadcasts, which entail more network means have less efficiency than link-level network reconfiguration.

To prevent from above drawbacks, an algorithm must be proposed which provide the fault tolerance without increasing the network congestion, traffic overload, much resource utilization. In this paper we have proposed an approach AARS in a wireless mesh network. The proposed approach is discussed with proper network parameters (i.e. throughput, packet ratio and packet drop ratio). The next section explains the AARS with diagrams.

\section{ADVANCED AUTONOMOUS NETWORK RECONFIGURATION SYSTEM (AARS)}

In this section, we have proposed an Advanced Autonomous Network Reconfiguration System (AARS) that allows a multi radio WMN (MR-WMN) to independently reconfigure its local network surroundings for real-time retrieval from link failures. AARS is fortified with a reconfiguration scheduling algorithm that 
recognizes local formation changes for the recovery. Briefly, AARS first pursuits for viable local configuration variations available around defective area, based on given channel and radio connotations. Then, by striking existing network specifications as constraints, AARS establish reconfiguration tactics that entail the minimum number of vagaries for the vigorous network settings. Autonomous Network Reconfiguration System also implements an observing protocol that permits a WMN to perform real-time disaster recovery in aggregation with the planning algorithm. The algorithm is explained by taking the three modules as depicted in Table 1 .

TABLE I. MODULE OF AARS

\section{Module 1: \\ Deployment Planning and Monitoring period}

In the Deployment Planning stage, planning is done to provide the shape to the network topology with $\mathrm{n}$ number of nodes (say 25), contiguous nodes are detached by certain distance (say 180m), each and every node is armed with altered number of radios, depending on its contiguity to a gateway. In monitoring period, AARS in every mesh node observers the quality of its retiring wireless associates and reports the outcomes to a gateway.

\section{Module 2:}

\section{Transmission and failure}

There are situations to rival real-time network actions. First, to generate user transportation from source node to destination node, i.e. multiple UDP streams between a gateway and arbitrarily chosen mesh node is familiarized with a packet dimension of 1000 bytes. Second, to create link failures, faults are inserted and lasts for a given failure period.

\section{Module 3:}

Failure detection and Reconfiguration

Once it notices a link failure, AARS in the sensor node(s) generates the formation of a cluster among native mesh routers that use a faulty channel, and one of the assemblage members is appointed as a lead via gateway using the eminent bully algorithm for organizing the reconfiguration. The lead node sends a planning-request communication to a gateway. Then, the gateway generates a reconfiguration proposal for the request. The gateway sends a reconfiguration strategy to the leader node and the group members. In the end, every node in the group executes the corresponding formation changes, if any, and re-establishes the connection and restart the process (starts re-sending the UDP packets).

Table 2 .

The corresponding pseudo code of Advanced Autonomous Reconfiguration System is depicted in

\section{TABLE II. ALGORITHM OF AARS}

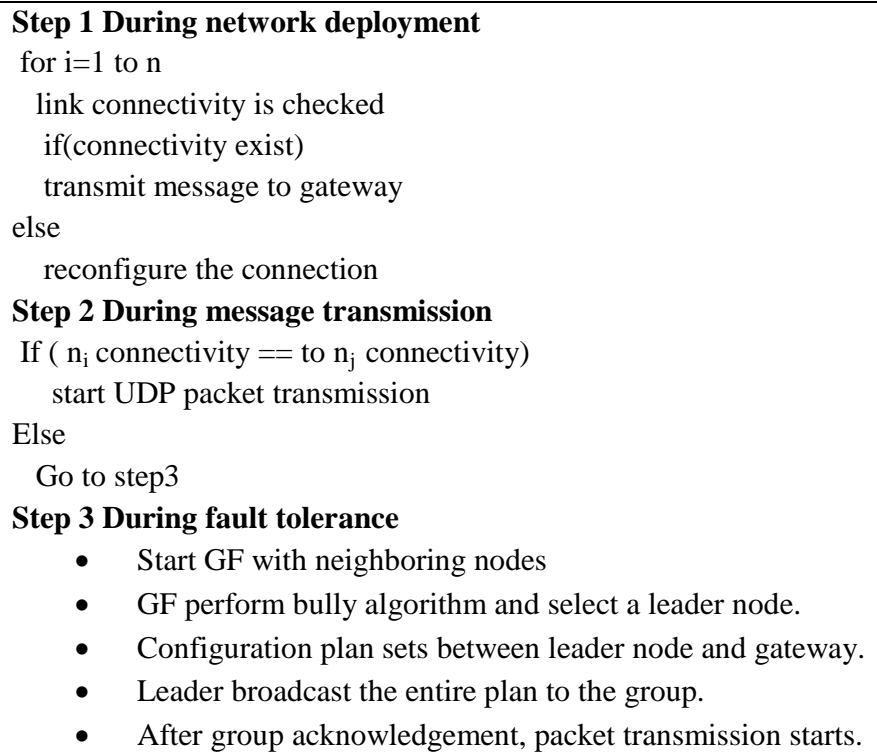

The following abbreviations are used in our algorithm i.e. $\mathrm{N}=$ number of nodes, $\mathrm{GF}=$ Group Formation, $\mathrm{Ni}=\mathrm{i}^{\text {th }}$ number of node, $\mathrm{Nj}=\mathrm{j}^{\text {th }}$ number of node. The execution of the AARS algorithm is explained in next section with their proper simulation graphs.

Step 1: In the initial phase of AARS, nodes are deployed in the WMN. Then, the gateway checks its connectivity with every node in mesh network as depicted in Figure 2. During transmission, phases, UDP packets of 1000 bytes are transmitted from source node to destination node. 


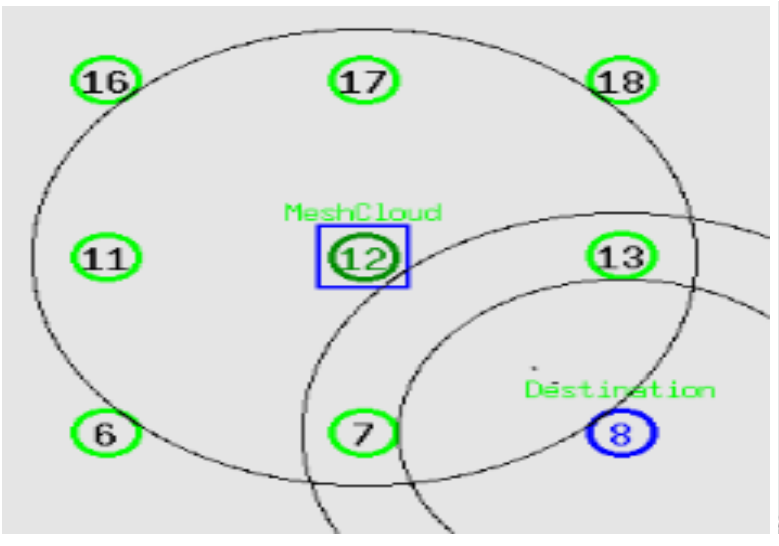

Fig. 2: Initial Phase

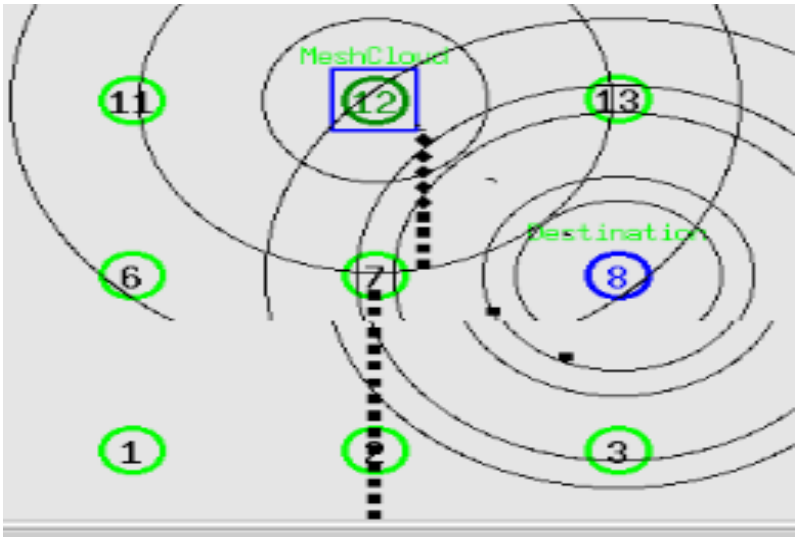

Fig. 3: Failure Occurence

Step 2: Figure 3 shows that after transmitting the packets, link failure occurs in the mesh that lasts for specific time.

Step 3: In Figure 4, AARS detects the link failure is the mesh network and triggers the detector node to form the group that includes the dead node. Then, gateway appointed one of the nodes in the group as a leader for reconfiguration, using bully algorithm. Leader and the gateway node reconfigure the faulty link and broadcast the plan to their group mates to re-establish the connection.

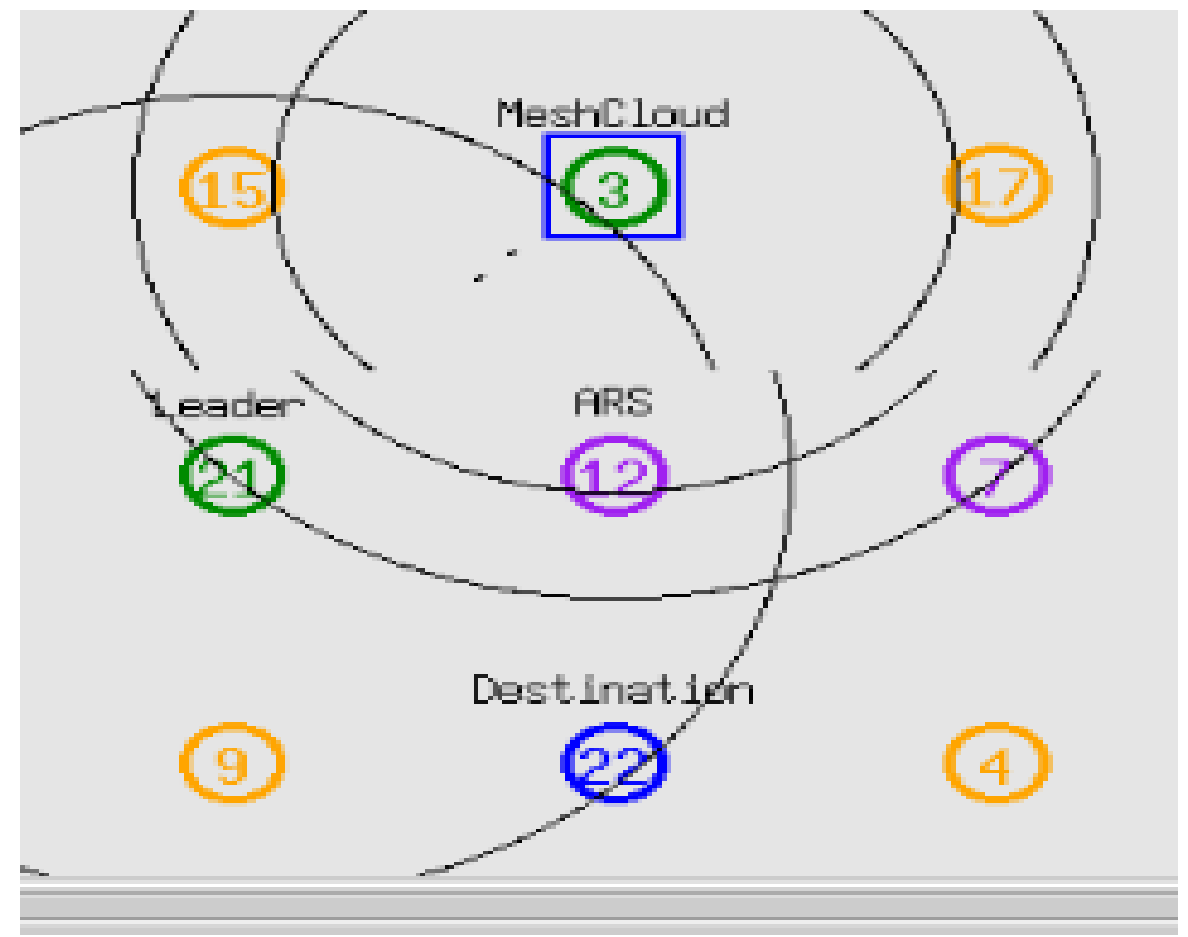

Fig. 4: Re-estaiblishment of Connection

\section{PERFORMANCE EVALUATION}

In order to evaluate algorithm, the AARS is simulate over NS2 simulator. The performance of the algorithm is measured in terms of throughput and packet ratio. The network size of the algorithm is shown in Table 3. 
Table III. NETWORK PARAMETERS

\begin{tabular}{|l|l|}
\hline Parameters & Size \\
\hline Number of nodes & 25 \\
\hline Network area & $180 \mathrm{~ms}$ \\
\hline MAC & 802.11 \\
\hline Start time & $0 \mathrm{~ms}$ \\
\hline Stop time & $15 \mathrm{~ms}$ \\
\hline Traffic Source & CBR \\
\hline Packet Size & 1000 bytes \\
\hline Antenna & Omni Antenna \\
\hline
\end{tabular}

Throughput is generally defined as the rate of packets transmission inside the network. In this paper, the throughput of the SRWMN and AARS as depicted in Figure 5 and Figure 6. As shown in Figure 5, throughput rate of SRWMN is $82.2 \%$ because as the number of faults inside the increases the algorithm is not able of recover and subsequently drops the packets. While in case of AARS throughput rate is $83.95 \%$, in case of fault occurrence reconfiguration of the faulty link takes place and recover the entire system without dropping the packets.

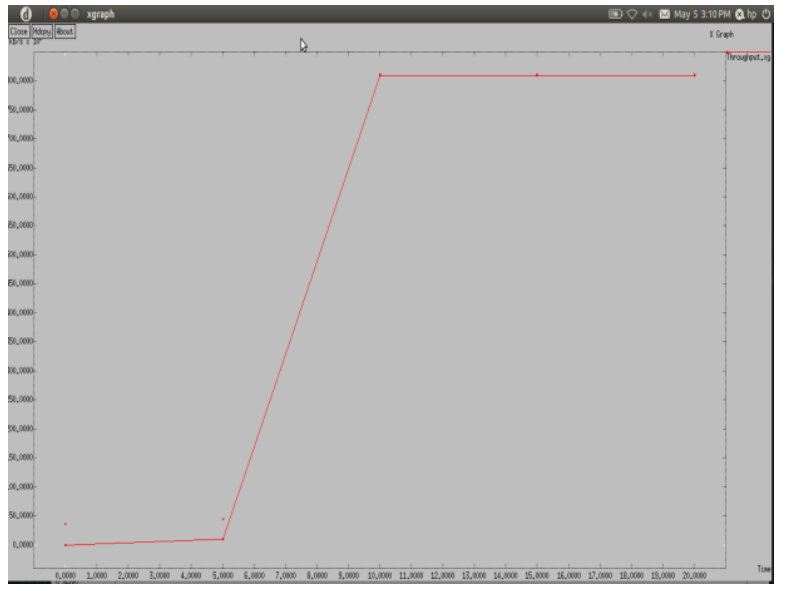

Fig. 5: Throughput of SRWMN

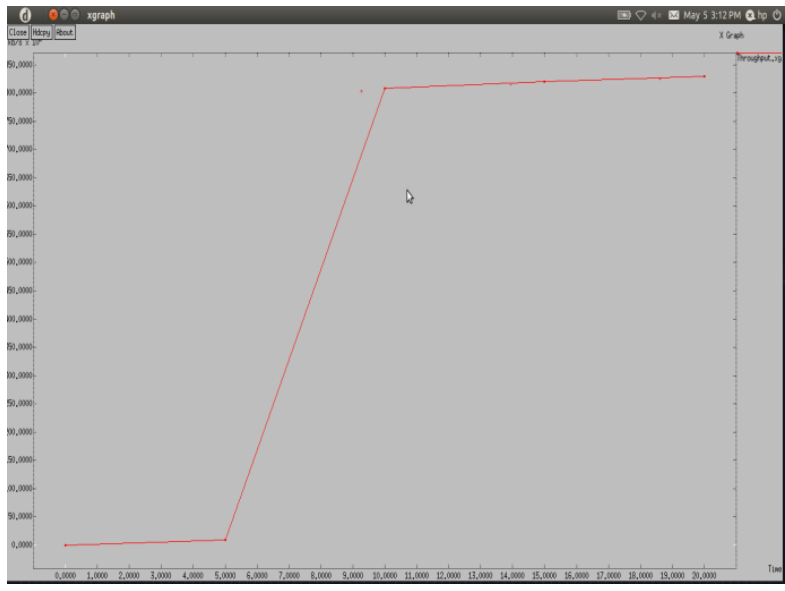

Fig. 6: Throughput of AARS

Packet Ratio is defined as the ratio of number of packets received to the number of packets transmitted. The graph of SRWMN as shown in Figure 7, in this graph during the fault occurrence packet ratio is decreased exponentially after the recovery phase the packet start receiving to the destination node. While in case of AARS during the fault occurrence packet ratio decreases initially but after that algorithm starts reconfiguration of the dead nodes and starts packet transmission. The graph of AARS algorithm is shown in Figure 8.

\section{CONCLUSION}

In this paper, an AARS algorithm is projected over WMN to recuperate from wireless link catastrophes. It generates an actual reconfiguration strategy which necessitates only indigenous network changes by using path and radio diversity. AARS effectually recognizes reconfiguration campaigns that please QoS constrictions, confessing up to two periods more drifts than static obligation, through QoS aware scheduling. Next, AARS allows for real-time fiasco detection and system reconfiguration, thus refining channel proficiency. This experimental appraisal on a Linux-based execution and ns2-based imitation has established the efficacy of AARS in recuperating from native link-failures and in adequate applications. 


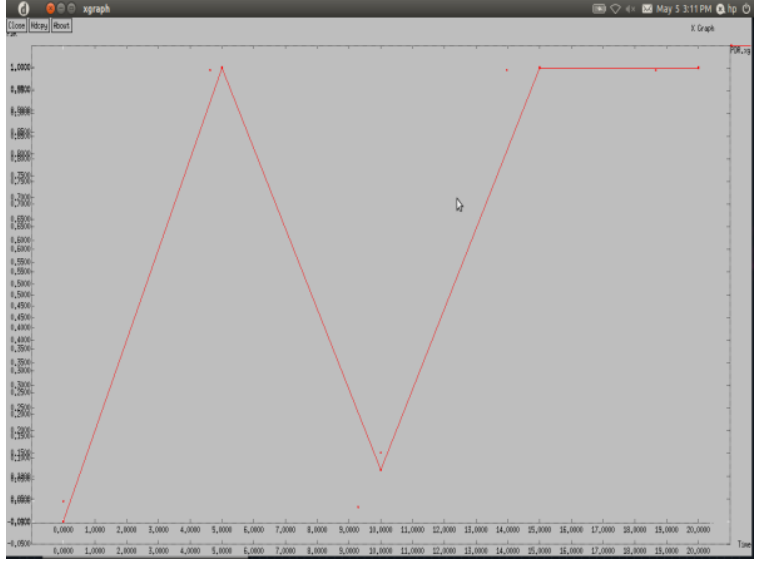

Fig. 7: Packet Ration of SRWMN

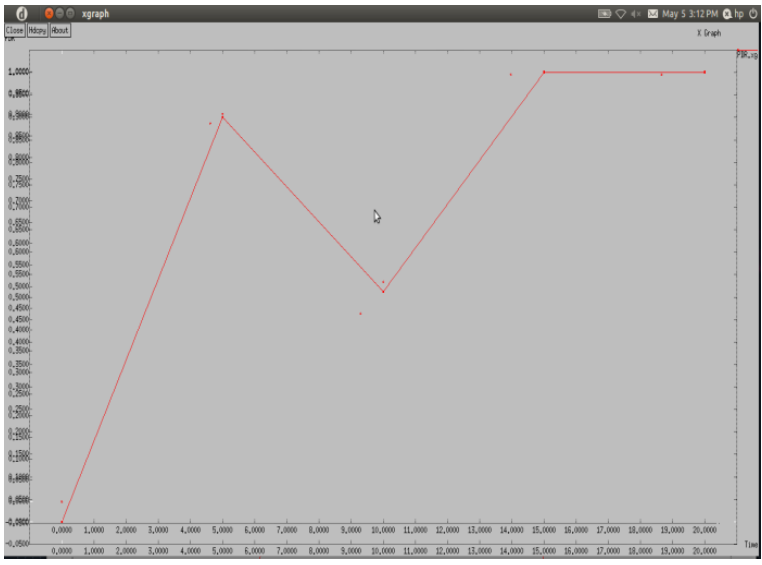

Fig. 8: Packet Ratio of AARS

\section{REFERENCES}

[1] F. Kaabi,S. Ghannay, and F. Filali, Channel Allocation and Routing in Wireless Mesh Networks: A survey and qualitative comparison between schemes, International Journal of Wireless and Mobile Networks(IJWMN),vol. 2 (1), 2010.

[2] Kyu-Han Kim, and Kang G. Shin, Self-Reconfigurable Wireless Mesh Networks, IEEE/ACM Transactions on Networking, vol. 19(2), 2011.

[3] Xiaoqin Chen, Haley M. Jones, and Dhammika Jayalath, Channel-Aware Routing in MANETs with Route Handoff, IEEE Transactions on Mobile Computing, vol. 10 (1), 2011.

[4] I. Akyildiz, X. Wang, and W. Wang, Wireless mesh networks: A survey, Computer Network, Vol. 47 (4), 2005 , pp $445-487$.

[5] A. P. Subramanian, H. Gupta, S. R. Das, and J. Cao, Minimum interference channel assignment in multi-radio wireless mesh networks, IEEE Transactions on Mobile Computing., vol. 7 (12), Dec. 2008, pp 1459-1473.

[6] J. David, R. Jayasingh, Novel Defense Scheme for Static and Dynamic Wireless Mess Network , International Journal of Scientific \& Engineering Research, vol. 3 (6), 2012.

[7] F. Kaabi1, S. Ghannay2, and F. Filali1, Channel Allocation and Routing in Wireless Mesh Networks, Journal of Network Security, vol.3 (1), 2011

[8] Sarasvathi, V., N. Ch SN Iyengar, and Snehanshu Saha, An efficient interference aware partially overlapping channel assignment and routing in wireless mesh networks, International Journal of Communication Networks and Information Security, vol. 6 (1), 2014. 